49 | 2018

Human-environment relationships in Siberia and Northeast China. Knowledge, rituals, mobility and politics among the Tungus peoples, followed by Varia

Braae Christel, Among Herders of Inner Mongolia. The Haslund-Christensen Collection at the National Museum of Denmark

Aarhus, Lancaster \& Oakville (CT), The Carlsberg Foundation's Nomad

Research Project/Aarhus University Press, 2017, 617 pages,

748 illustrations, ISBN 978-87-7934-395-5

Isabelle Charleux

\title{
OpenEdition
}

Journals

Édition électronique

URL : https://journals.openedition.org/emscat/3186

DOI : 10.4000/emscat.3186

ISSN : 2101-0013

Éditeur

Centre d'Etudes Mongoles \& Sibériennes / École Pratique des Hautes Études

Référence électronique

Isabelle Charleux, «Braae Christel, Among Herders of Inner Mongolia. The Haslund-Christensen Collection at the National Museum of Denmark», Études mongoles et sibériennes, centrasiatiques et tibétaines [En ligne], 49 | 2018, mis en ligne le 20 décembre 2018, consulté le 13 juillet 2021. URL : http://

journals.openedition.org/emscat/3186 ; DOI : https://doi.org/10.4000/emscat.3186

Ce document a été généré automatiquement le 13 juillet 2021.

() Tous droits réservés 


\section{Braae Christel, Among Herders of Inner Mongolia. The Haslund- Christensen Collection at the National Museum of Denmark}

Aarhus, Lancaster \& Oakville (CT), The Carlsberg Foundation's Nomad Research Project/Aarhus University Press, 2017, 617 pages, 748 illustrations, ISBN 978-87-7934-395-5

Isabelle Charleux

\section{RÉFÉRENCE}

Braae Christel, Among Herders of Inner Mongolia. The Haslund-Christensen Collection at the National Museum of Denmark, Aarhus, Lancaster \& Oakville (CT), The Carlsberg Foundation's Nomad Research Project/Aarhus University Press, 2017

1 Ce splendide et volumineux ouvrage sur la collection Haslund-Christensen du Musée national du Danemark est bien plus qu'un catalogue ; il constitue aujourd'hui l'étude la plus complète et la plus documentée en langue occidentale de la culture matérielle mongole du début du $\mathrm{xx}^{\mathrm{e}}$ siècle depuis le catalogue Die Mongolen, Haus der Kunst édité en 1989 par Walther Heissig et Claudius C. Müller (Heissig \& Müller 1989). La collection ethnographique de Copenhague - une des plus riches d'Europe et surtout une des mieux documentées - acquise principalement lors de deux expéditions danoises en Mongolie-Intérieure entre 1936 et 1939 était connue jusque-là principalement par deux publications de référence portant sur les costumes et sur les bijoux ${ }^{1}$.

2 La vie de Henning Haslund-Christensen (1896-1948), collectionneur, explorateur et écrivain, et l'histoire des expéditions danoises sont retracées dans le premier chapitre. Parti après une formation militaire avec Carl Krebs (1889-1971) établir une fermecolonie danoise dans le Nord-Ouest de la Mongolie en 1923 (actuelle province Hövsgöl), 
Haslund-Christensen se passionna pour les Mongols. Il eut l'occasion d'être recruté comme caravanier dans l'expédition sino-suédoise de l'explorateur géographe Sven Hedin (1865-1952) de 1927 à 1929, et fut ensuite invité à rester cinq semaines chez les Torgut des Tianshan (Xinjiang). Il y enregistra soixante chants «folkloriques » sur des cylindres de cire (conservés à Stockholm) et collecta des objets ethnographiques pour le Riksmuseet de Stockholm.

3 Le deuxième chapitre, "Scandinavian explorers in Mongolia » retrace la contribution scandinave aux connaissances sur la Mongolie (quelques autres objets mongols du musée viennent d'autres explorateurs danois). C. Braae décrit minutieusement le "réseau scandinave» en Mongolie et Mongolie-Intérieure aux $\mathrm{XIX}^{\mathrm{e}}$ et début du $\mathrm{xx}^{\mathrm{e}}$ siècle - explorateurs, missionnaires, aventuriers, scientifiques, hommes d'affaires, utopistes -, ainsi que leur réseau mongol, et s'attarde particulièrement sur les expéditions Hedin, qui inspirèrent celles de Haslund.

4 Le troisième chapitre est dédié aux deux expéditions scientifiques montées par Haslund, qui nécessitèrent d'âpres négociations avec les autorités japonaises. La première, en 1936-1937, était une mission de reconnaissance dans le Xing'an (Kingyan), le Barga (Baryu), la Mandchourie et chez les Solon et Dagur. La seconde, en 1938-1939 avec le linguiste Kaare Grønbech (1901-1957) et l'archéologue Werner Jacobson (1914-1979) sous les auspices de la Société royale de géographie du Danemark, avait trois objectifs: philologique, archéologique et ethnologique. Cette expédition scientifique à Hohhot, Kalgan et Chagan khuree sume (Cayan kürije süme, ancien temple devenu « résidence d'été » de Frans August Larson, 1870-1957) dans le Caqar en Mongolie-Intérieure, avec des sous-expéditions depuis Chagan khuree, bénéficia de l'aide de missionnaires suédois, et tout particulièrement de Georg Söderbom (1904-1973), collègue des expéditions Hedin. Il s'agissait de missions de sauvetage d'un patrimoine matériel et immatériel qui risquait de disparaître à jamais dans les conflits du début du $\mathrm{xx}^{\mathrm{e}}$ siècle. Alors que les expéditions précédentes collectaient au hasard, la collecte était ici méthodique. Les Mongols coopérèrent volontiers à la collecte comme à l'étude des objets, venaient de loin pour lui en apporter, et l'un de ses amis et assistants, le lama Lodai (un réfugié qalqa), réalisa à la demande d'Haslund un magnifique carnet de dessins aujourd'hui conservé au Musée national du Danemark.

C. Braae nous fait revivre les expéditions dans le détail, leur route, les moyens, l'organisation, les difficultés, leur lot d'espoir et de découragement, les aspirations scientifiques, les méthodes de collecte, l'équipement, et l'excitation des découvertes. Elle replace les expéditions danoises dans le contexte plus général des grandes expéditions commençant au $\mathrm{XIX}^{\mathrm{e}}$ siècle en lien avec la construction des musées ethnographiques en Europe.

6 Fin connaisseur de la culture et de la langue mongole, respectueux des coutumes, grand amateur de musique et de légendes qu'il notait au coin du feu, Haslund montra toujours une profonde admiration et beaucoup d'empathie pour ce peuple. Sa véritable passion était la musique ${ }^{2}$ et il chercha à établir des liens culturels entre les différents groupes mongols en comparant leurs chants et légendes. Il se voyait comme un écrivain et non comme un savant, mais sut s'entourer de chercheurs spécialisés pour étudier sa collection. Grâce à ses livres ${ }^{3}$, très populaires au Danemark et traduits dans de nombreuses langues, et ses conférences dans tout le pays, il devient une figure publique renommée en Suède et au Danemark et le départ de sa première expédition fut un événement national. Ses conférences étaient de «l'ethnographie vivante » combinant 
exposé ethnographique et performance musicale (une assistante habillée en mongole faisait semblant de jouer du luth et de chanter. Après la deuxième guerre mondiale, Haslund embarqua dans une nouvelle expédition en Asie centrale mais décéda à Kaboul en 1948 sans en récolter les fruits.

7 Pour reconstituer minutieusement la vie de cette petite société, l'auteur utilise une grande variété de sources - quelques 2500 documents d'archives dispersés (mémoires, lettres personnelles, rapports, factures, livres d'achat, journaux de voyage des différents membres du réseau) et des ouvrages publiés. L'ouvrage est illustré de nombreuses photographies d'époque longuement légendées, C. Braae ayant mené une véritable enquête pour identifier les protagonistes, lieux et monastères photographiés. Le lecteur y trouvera nombre d'informations et d'anecdotes fascinantes, sur la construction du télégraphe, les enregistrements effectués par Haslund dans une prison du Mandchoukouo, ou encore le mauvais sort attaché à un costume de chamane dont on se débarrasse en le désinfectant. Un certain nombre de questions demeurent, sur la raison pour laquelle Halsund n'a pas participé aux expéditions suivantes de Hedin, ou encore sur ses activités commerciales (et peut-être d'espionnage?) en Inde en 1929-1930.

8 Les objets acquis par les deux expéditions danoises étaient destinés au nouveau Musée national de Copenhague. Haslund et ses collègues achetèrent également des "doublons », c'est-à-dire un équivalent de chaque artefact, à la demande et avec le support financier de O.H. Bærentzen, ancien consul du Danemark à Nice. Ces «doublons» formèrent la "collection parisienne», qui fut transférée au Musée de l'Homme à Paris en $1946^{4}$.

9 Les objets du Musée national du Danemark ont été remarquablement étudiés depuis leur acquisition jusqu'à leur installation, comme $\mathrm{C}$. Braae le montre dans le quatrième chapitre. Haslund et ses collaborateurs notaient minutieusement l'origine, l'histoire et les circonstances d'acquisition de chaque objet dans des livres d'achat. Une fois arrivés au Danemark, les objets furent catalogués et classifiés par "tribu » mongole, avec un système de fiches comprenant leur photographie, puis furent incorporés dans des expositions ${ }^{5}$ et même mis en scène (des membres du musée se déguisant en chamanes ou mettant les costumes des «tribus » et jouant de la musique pour une performance intitulée « An evening in Mongolia »). Malgré la stricte séparation entre collectionneur et conservateur-savant qui avait cours, l'expertise d'Haslund fut utilisée dans la présentation des collections mongoles dans la nouvelle aile du musée en 1947.

10 La « collection Haslund-Christensen » compte un total de 2276 « objets » (en fait, plus de 3800 , puisque par exemple la yourte avec tout son mobilier compte pour une entrée). Aux artefacts des deux expéditions danoises s'ajoutent 224 objets entrés avant (voyageurs individuels) et après les expéditions (achats). La grande majorité sont des objets ethnographiques; mais la collection comprend également quelques 800 objets bouddhiques et chamaniques, dont 176 thang $k a$, environ 500 photos de HaslundChristensen et 500 de Grønbech (plus environ mille d'autres voyageurs), les carnets de voyage de Grønbech, 213 enregistrements audio, et 3000 spécimens archéologiques. Seuls quelques objets sont actuellement exposés dans une salle du musée. Des costumes sont exposés sur des mannequins dont le moulage du visage fut effectué par HaslundChristensen sur leurs anciens propriétaires. Grønbech avait également acheté 568 livres manuscrits et imprimés et cartes qui sont conservés à la Bibliothèque royale de Copenhague ${ }^{6}$. 
11 Les chapitres 5 à 14 présentent et analysent une sélection de 930 objets ethnographiques (dont 800 du Musée national du Danemark, le reste venant de la collection de Paris et d'autres donateurs danois) qui documentent la vie quotidienne d'une société pastorale en pleine mutation dans les années 1930. Ne figurent pas dans ce volume les costumes et les bijoux et coiffes, publiés séparément, et seule une petite sélection d'objets religieux est incluse. Les objets sont groupés par thèmes : campement et habitat (chapitre 5), production et consommation de nourriture (chapitre 6), élevage (chapitre 7), artisanat domestique (chapitre 8), chasse (chapitre 9), trois jeux virils (chapitre 10), jeux et jouets (chapitre 11), médecine traditionnelle (chapitre 12), possessions personnelles (chapitre 13) et comptabilité des caravanes et sceaux (chapitre 14). Les objets proviennent de dix-huit groupes mongols, principalement de Mongolie-Intérieure ainsi que de réfugiés qalqa venant de Mongolie.

12 Les introductions aux chapitres, basées sur les notes d'Haslund et de ses collègues d'expédition, les notes et publications d'autres voyageurs et des sources ethnographiques contemporaines, sont d'excellentes synthèses documentant les différents aspects de la vie quotidienne. Les notices reproduisent des notes des explorateurs («collector's note »), notamment la description et les histoires attachées aux objets et à leur utilisation, des informations sur les artisans, les prix parfois, la date, provenance et circonstances d'acquisition, le propriétaire précédent et des anecdotes. Plusieurs des fiches de Söderbom, avec les noms de l'objet écrits en chinois et en mongol ainsi qu'un dessin en montrant l'utilisation sont reproduites (pp. 270, 386, 428, 400 - dans cette dernière il s'excuse d'écrire au crayon car il est en prison, détenu en 1939 par les Japonais). Le catalogue est illustré de photographies d'époque, souvent mises en scène, tel Lodai se déguisant en lama jod (tib. gcod) (p. 213).

Le lecteur découvrira la richesse de la culture matérielle mongole - certes en partie produite par des artisans chinois -, avec de nombreuses variations selon les différents groupes («tribus »). Nombre de ces objets, notamment les ustensiles de cuisine, ne sont plus utilisés aujourd'hui. On y apprend par exemple qu'une liqueur médicinale chinoise empêche les pieds de sentir fort (p. 309) ; comment les caravaniers chinois font boire les Mongols pour qu'ils vendent tout ce qu'ils ont (p. 321); comment les marchands chinois truquent leurs balances (p. 424); comment traiter la colique du chameau (p. 406) et réhydrater leur nourriture en urinant dans leur gamelle, et de nombreux détails sur les techniques du corps (les bonnes manières d'uriner et de déféquer dans le Caqar, pp. 221-222).

14 C. Braae a fait le choix de laisser les transcriptions utilisées par Haslund et ses collaborateurs, mais les mongolisants rétabliront aisément la plupart des termes, et les linguistes devraient être intéressés par les terminologies et variantes dialectales.

Ce remarquable catalogue qui met en valeur la contribution des Danois aux études mongoles est une source essentielle pour les spécialistes de la culture matérielle mongole, du patrimoine et de la fondation des collections et musées ethnographiques en Europe. 


\section{BIBLIOGRAPHIE}

Boyer, M. [1952] 1995 Mongol Jewelry (Copenhague/Londres, The Carslberg's Foundation Nomad Research Project/Rhodos International Science and Art Publishers/Thames and Hudson).

Dupaigne, B. (éd.) 1983 Mongolie-Mongolie, traditions de la steppe (Paris, Musée de l'Homme, présentation des collections du Musée national d'Oulan-Bator et du Musée de l'Homme du 2 mars au 23 mai 1983).

Hansen, H. H. [1950] 1993 Mongolian Costumes (Copenhague/Londres, The Carslberg's Foundation Nomad Research Project/Rhodos International Science and Art Publishers/Thames and Hudson).

Haslund-Christensen, H. 1932 Jabonah (Copenhague, Gyldendalske Boghandel/Nordisk Forlag). 1934 Tents in Mongolia (Yabonah). Adventures and Experiences among the Nomads of Central Asia, traduit du danois par E. Sprigge \& C. Napier (Londres, Kegan Paul, Trench, Trubner \& Co.). 1935a Zajagan (Copenhague, Gyldendalske Boghandel/Nordisk Forlag). 1935b Men and Gods in Mongolia (Zayagan), traduit du danois par E. Sprigge \& C. Napier (Londres, Kegan Paul, Trench, Trubner \& Co.).

1945 Asiatiske strejftog (Copenhague, Gyldendalske Boghandel/ Nordisk Forlag).

1949 Mongolian Journey, traduit du danois par F. H. Lyon (Londres, Routledge \& Paul Kegan).

Heissig, W., assisté par Charles Bawden 1971 Catalogue of Mongol Books, Manuscripts and Xylographs (Copenhague, The Royal Library).

Heissig, W. \& C. C. Müller (éds) 1989 Die Mongolen, Haus der Kunst, I-II (Frankfurt/Innsbruck, Pinguin/Umschau).

\section{NOTES}

1. Boyer 1952 et Hansen 1950, tous deux réédités respectivement en 1995 et 1993.

2. Lors de sa première expédition Haslund partit dans le Xing'an avec un volumineux gramophone et 300 disques de laque dans 14 boîtes lourdement chargées. Les 113 chants enregistrés furent étudiés dans un volume de la Sino-Swedish Expedition (vol.21/8), mais il ne publia pas d'étude sur la musique enregistrée chez les Torgut en 1928-1929.

3. Notamment : Jabonah en 1932, traduit en anglais en 1934, sur ses premières années en Mongolie avec Krebs ; Zajagan en 1935, traduit en anglais la même année, sur sa participation à l'expédition Hedin chez les Torguts ; Asiatiske strejftog en 1945, traduit en anglais en 1949, sur ses expéditions (Haslund-Christensen 1932, 1934, 1935a, 1935b, 1945, 1949).

4. Voir le catalogue Mongolie-Mongolie, 1983 auquel ont contribué F. Aubin, L. Delaby et B. Dupaigne (Dupaigne 1983). Au Musée du Quai Branly, où elle se trouve depuis 2004, la collection est cataloguée sous le nom de "Mission Bærentzen », et comprend 249 objets (MH 46.42.1-MH 46.42.255).

5. En 1970, l'exposition «Buddha's Ways » à Copenhague présenta plus de 2000 objets de la deuxième expédition.

6. Ils sont catalogués dans Heissig (assisté par Charles Bawden) 1971. 


\section{AUTEURS}

\section{ISABELLE CHARLEUX}

GSRL, UMR 8582, CNRS - EPHE, PSL, Paris (France) 